\title{
ADVANCEMENTS IN OPEN GEOSPATIAL STANDARDS FOR PHOTOGRAMMETRY AND REMOTE SENSING FROM OGC
}

\author{
George Percivall $^{\mathrm{a}, *}$, Dr. Ingo Simonis ${ }^{\mathrm{b}}$ \\ a Open Geospatial Consortium, USA - gpercivall@opengeospatial.org \\ b Open Geospatial Consortium - Europe, United Kingdom - isimonis@opengeospatial.org
}

KEY WORDS: Geoprocessing, Remote Sensing, Open Standards, OGC

\begin{abstract}
:
The necessity of open standards for effective sharing and use of remote sensing continues to receive increasing emphasis in policies of agencies and projects around the world. Coordination on the development of open standards for geospatial information is a vital step to insure that the technical standards are ready to support the policy objectives. The mission of the Open Geospatial Consortium (OGC) is to advance development and use of international standards and supporting services that promote geospatial interoperability. To accomplish this mission, OGC serves as the global forum for the collaboration of geospatial data / solution providers and users. Photogrammetry and remote sensing are sources of the largest and most complex geospatial information. Some of the most mature OGC standards for remote sensing include the Sensor Web Enablement (SWE) standards, the Web Coverage Service (WCS) suite of standards, encodings such as NetCDF, GMLJP2 and GeoPackage, and the soon to be approved Discrete Global Grid Systems (DGGS) standard. In collaboration with ISPRS, OGC working with government, research and industrial organizations continue to advance the state of geospatial standards for full use of photogrammetry and remote sensing.
\end{abstract}

Reference: OGC Standards. http://www.opengeospatial.org/standards

\section{OPEN STANDARDS FOR REMOTE SENSING}

In the recently published "Common Framework for Earth Observation Data" the US President's Office of Science and Technology Policy (OSTP, 2016) declared that:

\begin{abstract}
"By standardizing the protocols for finding, accessing, and using Earth-observation data, the Common Framework will make it easier to obtain and assemble data from diverse sources for improved analysis, understanding, decision-making, community resilience, and commercial use."
\end{abstract}

The Framework was developed by OSTP interagency efforts led by the U.S. Group on Earth Observations. The Framework is intended to assist the data-management community in making decisions that will facilitate the greatest benefit to the United States and international community from the vast collective investment in Earth observations.

The OSTP Common Framework is a bold approach that builds and goes beyond previous policies applying open standards to Earth Observations including remote sensed data.

The GEOSS 10 Year Plan defined a bold vision to create a system of systems based on voluntary contributions to create a system of systems. Creating a system of systems for earth observations requires the use of open, consensus standards. With foresight, multiple organizations began been preparing for this challenge before GEOSS was defined. Standards and common practices for earth observations have been developed by organizations like ISPRS and the OGC. (GEOSS-OGC, 2010 .

The OGC (Open Geospatial Consortium) is an international, not for profit organization committed to making quality open standards for the global geospatial community. These standards are made through a consensus process and are freely available for anyone to use to improve sharing of the world's geospatial data. OGC standards are used in a wide variety of domains including Environment, Defense, Health, Agriculture, Meteorology, Sustainable Development and many more. OGC members come from government, commercial organizations, NGOs, academic and research organizations.

Open standards provide alternatives to vendor-controlled vertical integration of data collection, database management, analysis, portrayal and user interface. Once a radical idea, componentization is now reality. Small vendors, large vendors, solution providers and integrators can pick and choose components that are likely to "play well together" because of open standards. Small vendors can create components that make them "third party developers" to not one but several of the large vendors.

The OGC standards cover the functional needs for handling geographic information including remote sensing (Figure 1). For a full listing of OGC standards see (OGC Standards, 2016).

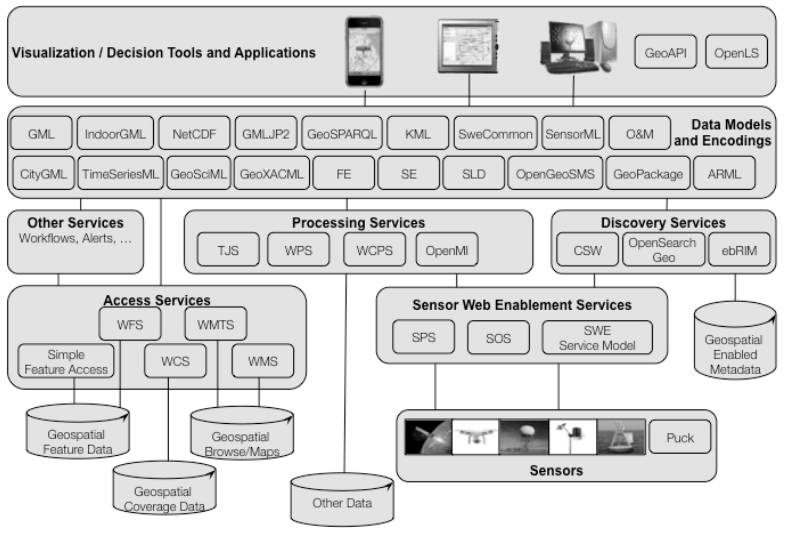

Figure 1. OGC Standards for Remote Sensing

* Corresponding author 
Figure 1 provides a summary of OGC Standards grouped into several categories:

- Observations from sensors are made interoperable through Sensor Web Enablement Services.

- Access Services provide protocols to make the variety of geographic information accessible in uniform fashion.

- Processing Services transform sensor observations and other information into information fit for the purpose of end users

- Discovery Services allow for users and services to discover resources of all types needed to meet the users needs.

- Other Services needed include workflow and security services.

- Data Models and Encodings are used across the multiple services.

\section{SENSOR WEBS FOR REMOTE SENSING}

\subsection{Remote Sensing acquisition as Observations}

The goal of OGC's Sensor Web Enablement (SWE, 2013) is to enable all types of Web and/or Internet-accessible sensors, instruments, and imaging devices to be accessible and, where applicable, controllable via the Web. SWE provides the standards foundation for "plug-and-play" Web-based sensor networks.

The OGC Sensor Observation Service (SOS) Implementation Standard defines a web service interface for requesting, filtering, and retrieving observations and sensor system information. Observations may be from in-situ sensors (e.g., water monitoring devices) or dynamic sensors (e.g., imagers on Earth-observation satellites).

The OGC Sensor Planning Service (SPS) Implementation Standard defines an interface to task sensors or models. Using SPS, sensors can be reprogrammed or calibrated, sensor missions can be started or changed, simulation models executed and controlled. The feasibility of a tasking request can be checked and alternatives may be provided. The OGC SPS Earth Observation Satellite Tasking Extension supports the programming process of Earth Observation (EO) sensor systems used by many satellite data providers.

The OGC Observations \& Measurements (O\&M) standard, also published as ISO 19156, defines a conceptual schema for observations, and for features involved in sampling when making observations. An observation is an act at a discrete instant or period, through which a number or term is assigned to a phenomenon using a procedure, such as a sensor, instrument, or algorithm.

The OGC Sensor Model Language (SensorML) Implementation Standard provides a framework within which the geometric, dynamic, and observational characteristics of sensors and sensor systems can be defined. SensorML enables robust definitions of sensor models for providing geolocation of observations from remote sensors. Different mathematical models can be designed to define a sample location within a variety of coordinate systems, including the local sensor frame, the local frame for the associated platform, or a geographic coordinate reference frame

The SWE Common Data Model Encoding Standard defines low-level data models for exchanging sensor related data between nodes of the SWE framework. These models allow applications and/or servers to structure, encode and transmit sensor datasets in a self-describing and semantically enabled way.

\subsection{Maturity of SWE}

An assessment of the maturity of implementations of OGC SWE standards was recently undertaken (Percivall, 2103). Beginning in 2001, there have been numerous implementations based on the SWE standards. The status as of the assessment in 2013 of the maturity of SWE implementation can be summarized as follows:

- SWE Version 1 standards are mature specifications with approved OGC compliance test suites and implementations. There are tens of independent implementations of the standards that have been deployed in operational systems. SWE version 1 is at Technology Readiness Level 9 - the highest level - and can confidently be mandated in system procurements.

- SWE Version 2 standards are in-process to be approved in the first half of this year (2013) [and were subsequently approved]. Building upon Version 1, the Version 2 specs have improved designs and additional functionality. Independent implementations have been developed and tested. Compliance tests are being finalized. SWE Version 2 is at Technology Readiness level 6 and has emerged for consideration in systems currently under development.

- Enhancements and coordination of the SWE standards are in a variety of states. In the OGC Interoperability Program initiatives, SWE has been used as a basis for a variety of topics: Workflows including SWE; Secure Sensor Web; Events and SWE; JPIP Streaming; Full Motion Video; Moving Object Indicators; GPS ephemeris/data, and more.

The SWE framework provides significant benefits for supporting the integration and fusion of a wide variety of assets, and readily enables a system that is able to sense and react to threats or opportunities. Very capable software components exist for supporting development of SWE implementations.

\section{COVERAGES AND BIG GEOSPATIAL DATA}

Coverages are a conceptual schema defined in OGC Abstract Specification, Topic 6 (OGC Coverages, 2007). Examples of coverages include rasters, triangulated irregular networks, point coverages and polygon coverages. Coverages are the prevailing data structures in a number of application areas, such as remote sensing, meteorology and mapping of bathymetry, elevation, soil and vegetation. For more information, see

The OGC Web Coverage Service (WCS) offers multidimensional coverage data for access over the Internet. The WCS Suite of standards (Figure 1) includes the WCS Core standard along with extensions, applications profiles and related encoding schemas. WCS extension standards add further functionality to the core; some of these are required in addition to the core to obtain a complete implementation. The core does not prescribe support for any particular coverage encoding format. WCS extensions specifying use of data encoding formats in the context of WCS are designed in a way that the GML coverage information contents specified in this core is 
consistent with the contents of an encoded coverage. For more information see (WCS, 2106)

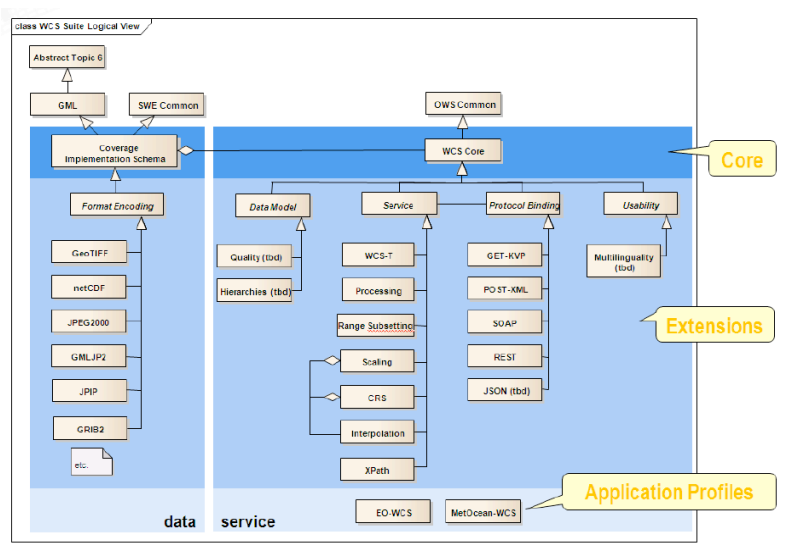

Figure 2. Web Coverage Service - Suite of Standards

The Coverage Implementation Schema (as shown in Figure 2) is a specification that is in the final stages of the OGC adoption process. (CIS was formerly known as GMLCOV). CIS specifies the OGC coverage model by establishing a concrete, interoperable, conformance-testable coverage structure. It is based on the abstract concepts of OGC Abstract Topic 6 (which is identical to ISO 19123) which specifies an abstract model which is not per se interoperable - in other words, many different and incompatible implementations of the abstract model are possible. CIS, on the other hand, is interoperable in the sense that coverages can be conformance tested, regardless of their data format encoding, down to the level of single "pixels" or "voxels".

For more information see (CIS, 2016)

Coverages can be encoded in any suitable format (such as GML, JSON, GeoTIFF or NetCDF) and can be partitioned, e.g., for a time-interleaved representation. Coverages are independent from service definitions and, therefore, can be accessed through a variety of OGC services types, such as WCS. The coverage structure can serve a wide range of coverage application domains, thereby contributing to harmonization and interoperability between and across these domains.

The OGC Web Coverage Processing Service (WCPS) defines a language for retrieval and processing of multi-dimensional geospatial coverages. Services implementing this language provide access to original or derived sets of geospatial coverage information, in forms that are useful for client-side rendering, input into scientific models, and other client applications. WCPS relies on the coverage model as defined in OGC Abstract Specification Topic 6 and the WCS standard. The WCPS language is independent from any particular request and response encoding, as no concrete request/response protocol is specified by WCPS.

For more information see (WCPS, 2016)

Use of WCS with array databases and Big Data applications is a rich and innovative area of application. Two excellent examples are Geoscience Australia Data Cube (Purss, 2015) and EarthServer (Baumann, 2016) projects.

\section{OGC ENCODINGS FOR REMOTE SENSED DATA}

\subsection{NetCDF}

netCDF is a set of software libraries and self-describing, machine-independent data formats that support the creation, access, and sharing of array-oriented scientific data. The conventions for climate and forecast (CF) metadata are designed to promote the processing and sharing of netCDF files. The conventions define metadata that provide a definitive description of what the data represents, and the spatial and temporal properties of the data.

The OGC CF-netCDF standard consists of a suite of standards that support encoding of digital geospatial information representing space/time-varying phenomena. Although it was originally developed for the Earth science community, netCDF can be used to communicate and store a wide variety of multidimensional data. The netCDF data model and encodings are particularly well suited to providing data in forms familiar to atmospheric and oceanic scientists, specifically, as sets of related arrays.

The CF-netCDF Core and Extensions Primer provides an overview of the many possible components of the CF-netCDF suite and explains how those components fit together into a coherent whole (Figure 3).

For more information see (NetCDF, 2016)

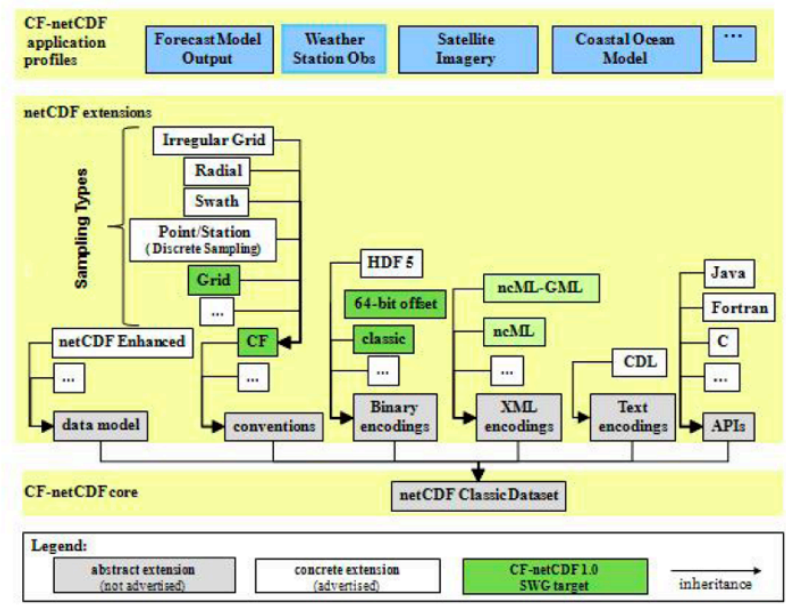

Figure 3. CF-NetCDF Specification hierarchy

\subsection{GMLJP2}

The OGC GML in JPEG 2000 for Geographic Imagery (GMLJP2) Encoding Standard defines the means by which the OGC Geography Markup Language (GML) Standard is used within JPEG 2000 images for geographic imagery. The standard also provides packaging mechanisms for including GML within JPEG 2000 data files and specific GML application schemas to support the encoding of images within JPEG 2000 data files. JPEG 2000 is a wavelet-based image compression standard that provides the ability to include XML data for description of the image within the JPEG 2000 data file.

For more information see (GMLJP2, 2016) 


\subsection{GeoPackage}

The OGC GeoPackage Encoding Standard defines a platformindependent SQLite database file that may contain:

- Vector geospatial features

- Tile matrix sets of imagery and raster maps at various scales

- Metadata

Since a GeoPackage is a database, it supports direct use, meaning that its data can be accessed and updated in a "native" storage format without intermediate format translations. GeoPackages are interoperable across all enterprise and personal computing environments, and are particularly useful on mobile devices like cell phones and tablets in communications environments with limited connectivity and bandwidth. This OGC Encoding Standard defines the schema for a GeoPackage, including table definitions, integrity assertions, format limitations, and content constraints. The allowable content of a GeoPackage is entirely defined in this specification.

For more information see (GeoPackage, 2016)

\section{DISCRETE GLOBAL GRID SYSTEMS}

The OGC Discrete Global Grid Systems (DGGS) candidate standard (DGGS, 2016) defines a set of rules for defining highly efficient architectures for spatial data storage and analytics. The goal of DGGS is to enable rapid integration of spatial data without the difficulties of working with legacy coordinate systems. DGGSs represent the Earth as sequences of cell tessellations each with global coverage and with progressively finer spatial resolution. Individual observations can be assigned to a cell corresponding to both the position and size of the phenomenon being observed. DGGS come with a standard set of functional algorithms that enable rapid data analysis of very large numbers of cells. The OGC DGGS standard supports the specification of standardized DGGS infrastructures that enable the integrated analysis of very large, multi-source, multiresolution, multi-dimensional, distributed geospatial data.

See:

http://www.opengeospatial.org/pressroom/pressreleases/2349
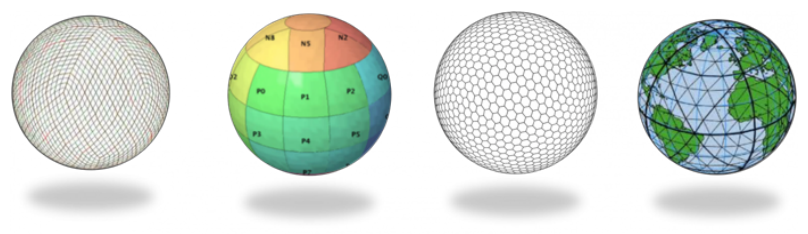

Figure 4. Discrete Global Grid System Examples

Figure 4 provides four examples of DGGS: 1) ISEA Triangular Grid, 2) SCENZ-Grid, 3) ISEA Hexagonal Grid, and 4) Quaternary Triangular Mesh global grid (fourth graphic used by permission of Geoffrey Dutton).

There is explosive growth in both the variety and the volume of spatial data and processing resources, along with a growing understanding of the tremendous benefit that can be derived from enabling interoperability between them. On the other side of this deluge of spatial content is a growing demand by decision-makers for a participatory environment where content can be accessed directly from diverse contributors and used with other content without reliance on time-consuming and costly geographic transformation processes.

One of the core contributions of a DGGS is geospatial data fusion on demand. In a multiple provider environment, fusion is only possible with an information system architecture based upon open standards. The candidate OGC DGGS Core Standard enables interoperability within and between different Discrete Global Grid Systems and it promotes reusability, knowledge exchange, and choices.

\section{STANDARDS COORDINATION}

\subsection{The OGC approach to open standards}

The OGC provides a collaborative, consensus process for developing, approving and maintaining open, international standards that enable the modelling, sharing, and access to any location enabled content or service. "International standards" are those adopted by an international standardizing/standards organization, often referred to as an SDO, and made available to the public. More specifically, the OGC is a Voluntary Consensus Standards Organization. "Voluntary consensus standards bodies" are domestic or international organizations which plan, develop, establish, or coordinate voluntary consensus standards using agreed-upon procedures.

According to the US Government OMB Circular A-119 (OMB, 1998), a voluntary consensus standards body is defined by the following attributes:

- Openness.

- Balance of interest.

- Due process.

- An appeals process.

- Consensus

Consensus in (OMB 1998) is defined as general agreement, but not necessarily unanimity, and includes a process for attempting to resolve objections by interested parties, as long as all comments have been fairly considered, each objector is advised of the disposition of his or her objection(s) and the reasons why, and the consensus body members are given an opportunity to change their votes after reviewing the comments.

The above-stated characteristics are fundamental to the OGC policies and procedures framework and guidelines for the collaborative and consensus development of OGC standards. While the OMB circular definition suggests that consensus is a separate attribute, in reality how the organization views and operates with regard to openness, balance of interest, due process, and an appeals process are all fundamental aspects of an open consensus process.

\subsection{ISPRS - OGC coordination}

Geospatial information is a cross-cutting requirement critical to many different domains; therefore OGC is aligned with the missions of many different organizations. In order to advance our common interests OGC actively collaborates with other organizations. Many of these organizations collaborate with us on standards development, particularly when an interoperability solution depends on consistency between standards from different standards development organizations. Standards organizations look to the OGC for advice on how to encode 
geolocation in their standards and we look to them to learn what we must do to achieve harmonization across the standards stack.

OGC and ISPRS work jointly in order that research on photogrammetric and remote sensing informs development of open interoperability standards for global benefit.

ISPRS and OGC have identified these areas of common interest

- 3D Data Visualization, CityGML, IndoorGML

- Web Services for Remote Sensed data

- $\quad$ Sensor Web and IoT

- Discrete Global Grid Systems (DGGS)

- $\quad$ ISPRS IJGI - OGC Special Issue

- $\quad$ ASPRS Manual of Remote Sensing, version $4^{\text {th }}$

Further coordination between ISPRS and OGC representatives is anticipated during the ISPRS Congress in 2016

\section{REFERENCES}

Baumann, 2016. Big Data Analytics for Earth Sciences: the EarthServer approach, Peter Baumann, et.al. International Journal of Digital Earth, Vol. 9, Issue 1, 2016 DOI:10.1080/17538947.2014.1003106

CIS, 2016. Call for comments on OGC Coverage Implementation Schema. Accessed on 17 April 2016. http://www.opengeospatial.org/pressroom/pressreleases/2345

DGGS, 2016. Call for comments on The OGC Discrete Global Grid Systems (DGGS) candidate standard. Accessed on 17 April 2016 .

http://www.opengeospatial.org/pressroom/pressreleases/2349

GeoPackage 2016. The OGC GeoPackage Encoding Standard. $\begin{array}{lllll}\text { Accessed on } & 17 & & \end{array}$ http://www.opengeospatial.org/standards/geopackage

GEOSS-OGC, 2010. OGC Statement to the GEOSS Beijing Ministerial Summit, 5th of November 2010. https://www.earthobservations.org/documents/ministerial/beijin g/statements/bms_OGC_statement.pdf
GMLJP2, 2016. The OGC GML in JPEG 2000 for Geographic Imagery (GMLJP2) Encoding Standard. Accessed on 17 April 2016. http://www.opengeospatial.org/standards/gmljp2

NetCDF, 2016. OGC CF-netCDF standard. Accessed on 17 April 2016. http://www.opengeospatial.org/standards/netcdf

OGC Coverages, 2007. OGC Abstract Specification, Topic 6 Schema for coverage geometry and functions, OGC Document 07-011, 2007-12-28. (Content identical to ISO 19123) http://portal.opengeospatial.org/files/?artifact_id=19820

OGC Standards, 2016. http://www.opengeospatial.org/docs/is

OMB, 1998. "Federal Participation in the Development and Use of Voluntary Consensus Standards and in Conformity Assessment Activities", US Government OMB Circular A-119, 1998. http://www.nist.gov/standardsgov/omba119.cfm\#4

OSTP, 2016. "Common Framework for Earth Observation Data," US President's Office of Science and Technology Policy (OSTP), March 2016. https://www.whitehouse.gov/sites/whitehouse.gov/files/docume nts/Common_Framework_for_Earth_Observation\%20Data_032 316.pdf

Percivall, 2013. OGC SWE Implementation Maturity Engineering Report, OGC Document 13-032, George Percivall editor, 2013-09-11

https://portal.opengeospatial.org/files/?artifact_id=53823

Purss, 2015. Unlocking the Australian Landsat Archive - From dark data to High Performance Data infrastructures, Matthew B.J. Purss, et.al., GeoResJ, Volume 6, June 2015, Pages 135140.

http://www.sciencedirect.com/science/article/pii/S22142428150 00182

SWE, 2013. OGC Sensor Web Enablement: Overview And High Level Architecture, OGC White Paper, document 07165r1, Carl Reed, Mike Botts, George Percivall, John Davidson, 2013-04-02. http://docs.opengeospatial.org/wp/07-165r1/

WCPS, 2016. The Web Coverage Processing Service (WCPS) standard. Accessed on 17 April 2016. http://www.opengeospatial.org/standards/wcps

WCS, 2016. The OGC Web Coverage Service (WCS) suite of standards. Accessed on 17 April 2016. http://www.opengeospatial.org/standards/wcs 